\title{
Centro de Estudios en Vivienda de Interés Social - CENVIS, Pasado, Presente y Futuro
}

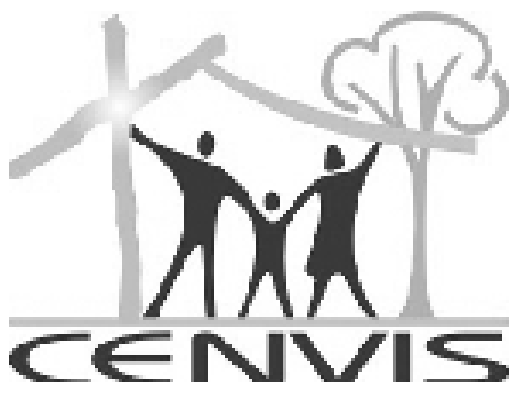

\section{Preparado por la Dirección del CENVIS con la participación del Ingeniero Edgar Sánchez esanchez@uniminuto.edu}

La Corporación Minuto de Dios como entidad comprometida a la luz del evangelio con el desarrollo integral de la persona humana y de comunidades marginadas, tanto rurales como urbanas, a través del programa de ingeniería civil de la Corporación Universitaria Minuto de Dios, denominado en un comienzo como ingeniería para el desarrollo social y desde entonces encargado de capacitar profesionales comprometidos de modo especial en el trabajo con las comunidades necesitadas, estableció en 1998 un acuerdo de cooperación con el Ministerio de la Construcción de la República de Cuba, con el fin de asesorar a la Facultad de Ingeniería en la creación de un centro de estudios en vivienda.

Producto de este acuerdo se designó al Ingeniero Nelson Navarro, profesor y especialista del Centro Técnico de Viviendas y Urbanismo del Instituto Nacional de la Vivienda de Cuba, para que trabajase con los docentes y directivos de la Corporación Universitaria.

Resultado del trabajo conjunto entre el profesor Nelson Navarro, docentes y alumnos se presento en abril de 1999 a la Facultad de Ingeniería la propuesta para la creación del Centro de Estudios en Vivienda de Interés Social - CENVIS

El primer proyecto de importancia para el CENVIS fue desarrollado en el proceso de reconstrucción de Calarcá, preparando perfiles de proyectos, conferencias sobre diseño estructural-constructivo a profesionales de la construcción y afines.

Posteriormente, el CENVIS trabajó en mejoramiento de entorno y vivienda, a través de la ejecución de convenios y contratos con entidades del orden nacional y distrital, realizando obras de urbanismo externo y equipamientos en urbanizaciones, con lo que podríamos enmarcar esta gestión como parte de la línea de investigación de mejoramiento del hábitat. Dentro de estos proyectos se podrían mencionar: 
- Proyecto Empleo en Acción - Plan Colombia.

- Proyecto de Acompañamiento Social y Técnico a la comunidad de la Ciudadela Nuevo Usme - METROVIVIENDA.

- Proyecto Interventoría Técnica Social y Ambiental del Plan Piloto del Proyecto Obras con Participación Ciudadana - OPC - DAACD.

\section{Proyecto Empleo en Acción - Plan Colombia} (2001 - 2003).

Éste proyecto contempló el desarrollo de actividades conjuntas entre el Departamento Administrativo de la Presidencia de la República - Fondo de Inversión para la Paz - DAPR-FIP y el Departamento Administrativo de Acción Comunal Distrital - DAACD para la construcción de obras de infraestructura en Bogotá, las cuales fueron ejecutadas por Juntas de Acción Comunal y Organizaciones Comunitarias. Dentro de dichas actividades se encuentran la adquisición de materiales y la selección y cancelación de aportes al personal de mano de obra no calificada de cada uno de lo proyectos, entre otras.

\section{Resultados:}

- 52 Proyectos en barrios marginales de Bogotá (Salones Comunales, Zonas verdes, Vías peatonales).

- Más de 700 millones de pesos administrados.

- Más de 600 empleos transitorios generados.

- Presencia en 11 Localidades con alto índice de pobreza.

Proyecto de Acompañamiento Social y Técnico a la comunidad de la Ciudadela Nuevo Usme - METROVIVIENDA (2003 - 2004).

Con la ejecución de éste proyecto se logró facilitar el desarrollo sostenible de la comunidad habitante de la Ciudadela Nuevo Usme y su entorno físico. Para tal fin, se propuso llevó a cabo un acompañamiento integral durante dos años a las familias habitantes de la Ciudadela Nuevo Usme, en Bogotá D.C.

Componentes del proyecto:

1. Asistencia Técnica en el desarrollo progresivo de las viviendas.

2. Acompañamiento en el proceso de organización comunitaria.

Proyecto Interventoría Técnica Social y Ambiental del Plan Piloto del Proyecto Obras con Participación Ciudadana - OPC - DAACD (2004 - 2005).
Se basó en el fortalecimiento de la organización y participación social y ciudadana a través del desarrollo de obras de interés comunitario, involucrando a las comunidades organizadas y la administración Distrital en todas las fases del proceso.

\section{Resultados:}

- 10 Proyectos supervisados (vías peatonales, parques y adecuación de consultorios médicos). - Presencia en 5 localidades con alto índice de pobreza.

Posteriormente se inicia una etapa en formulación de líneas de investigación y generación de un plan estratégico, los cuales se encuentran actualmente organizados de la siguiente manera:

\section{Mejoramiento del hábitat}

2. Infraestructura básica.

3. Tecnologías aplicadas a VIS.

4. Prevención de riesgos.

\section{Mejoramiento del Hábitat.}

Corresponde a las acciones que conduzcan a mejorar las condiciones físicas, ambientales y funcionales de la vivienda y el hábitat, en un marco de respeto por las normas de construcción, ambientales y de urbanismo, aportando al desarrollo integral y sostenible de los habitantes de los sectores más deprimidos de la ciudad.

\section{Infraestructura básica.}

Se refiere a la intervención sobre el equipamiento elemental de cualquier conglomerado habitacional (redes de servicios públicos, vías, equipamiento social y saneamiento básico), buscando generar unas condiciones mínimas de habitabilidad y de interacción social.

\section{Tecnologías de construcción en VIS.}

Se refiere a la investigación en materiales y sistemas de construcción, tanto tradicionales como innovadores, aplicados especialmente en la construcción de Vivienda de Interés Social con un criterio de desarrollo sostenible.

\section{Prevención de Riesgos.}

Se refiere a la sensibilización, capacitación y asesoría a la población más vulnerable, localizada en los asentamientos de origen ilegal, en la identificación y control de riesgos presentes en sus viviendas y entorno, causados por fenómenos naturales o antrópicos, de manera que se proteja su vida y su patrimonio.

Problema planteado 
En la actualidad el CENVIS concibe la problemática de vivienda de interés social bajo causas claras como son:

- El desplazamiento de familias de las áreas rurales a los cascos urbanos de las grandes ciudades, producto de la búsqueda de mejores oportunidades, o consecuencia del conflicto armado, plantea unos retos inmensos para las ciudades grandes y medianas.

- El $72 \%$ de los 42 millones de habitantes vive en las zonas urbanas, entendidas tanto las cabeceras como los demás centros poblacionales que se desarrollan en torno a ellas; queda un $28 \%$ en las zonas rurales o aquellas con un bajo nivel de urbanización.

- Los efectos son claramente predecibles en cuanto a pobreza, el suministro de servicios públicos domiciliarios, la capacidad para proveer los empleos suficientes, los altos precios de la tierra urbanizable, la ocupación informal del suelo, hacinamientos y problemas de salud pública en general, manifestaciones propias de los asentamientos precarios, agravan los problemas de tipo administrativo, fiscal y de planeación por todos conocidos.

- El enfoque tradicional de la política de vivienda, que se dirige a la reactivación económica y a la generación de ingresos, ha originado dos problemas que violan este derecho. Identificar que la solución es la construcción y compra de vivienda, y por lo tanto la orientación de esfuerzos y recursos estatales hacia aquellos grupos de ingresos más elevados que están en capacidad de comprar vivienda, dejando a un lado a las personas más pobres y vulnerables. Como resultado, estas personas no tienen otra opción que construir su vivienda en asentamientos subnormales.

- El sistema de financiamiento a largo plazo para la adquisición de vivienda en Colombia no permite el acceso de la población más pobre y casi siempre vinculada al sector informal de la economía y el auto empleo, lo que constituye un factor de discriminación.

- El acceso a los títulos de propiedad cuando se trata de asentamientos ilegales que se han establecido y crecen sobre la base de procesos de expropiación seguida de tierras (o invasión), no siempre de propiedad privada y casi siempre perteneciente a las reservas públicas, o a través de procesos irregulares de adquisición con urbanizadores piratas. Este problema constituye uno de los grandes retrasos en Colombia, porque la falta de solución jurídica y legal y la carencia de título impiden entrar de lleno en programas de probada viabilidad como es el mejoramiento de vivienda y el mejoramiento integral de barrios.

- En materia de vivienda se sigue hablando solo de la vivienda, cuando deberíamos haber avanzado ya hacia el concepto de hábitat para la vivienda, logrando combinar lo privado, lo íntimo, con lo colectivo, con lo social, y a ello darle legitimidad como derecho.

- Construir ciudad de manera equitativa, no excluyente, significa entonces distribuir de manera justa también los costos de urbanismo, y asegurar que todos reciben vías, infraestructura y equipamientos de acuerdo con las necesidades que marcan parámetros tan reales como la densidad de la población.

- A modo de conclusión se puede afirmar, en el marco de lo expuesto, que no ha existido un manejo adecuado de los procesos de desarrollo urbano, particularmente a propósito del desarrollo de la vivienda y no se conciben soluciones de largo plazo que den respuestas, no a situaciones coyunturales y a problemas de urgencia, sino a problemas de fondo que permitan ordenar el desarrollo urbano, y detrás de él la vivienda, la provisión de servicios públicos, vías, equipamientos y los demás valores que contribuyen a mejorar la calidad de vida de las personas.

\section{Objetivo General}

En la actualidad el CENVIS concibe como objetivo general de su labor el "Diseñar y desarrollar estrategias y líneas de investigación que permitan implementar y ejecutar metodologías que contribuyan al mejoramiento de las condiciones de vivienda y hábitat en las zonas en donde se localizan los asentamientos humanos más vulnerables de nuestras ciudades".

\section{Líneas de Acción}

Dicha labor está enmarcada en cinco líneas de acción, consideradas como prioritarias a fin de lograr el objetivo planteado y son:

\section{Vivienda nueva.}

2. Mejoramiento de vivienda.

3. Construcción en sitio propio.

4. Arriendo protegido (transición).

\section{Viviendas transitorias.}




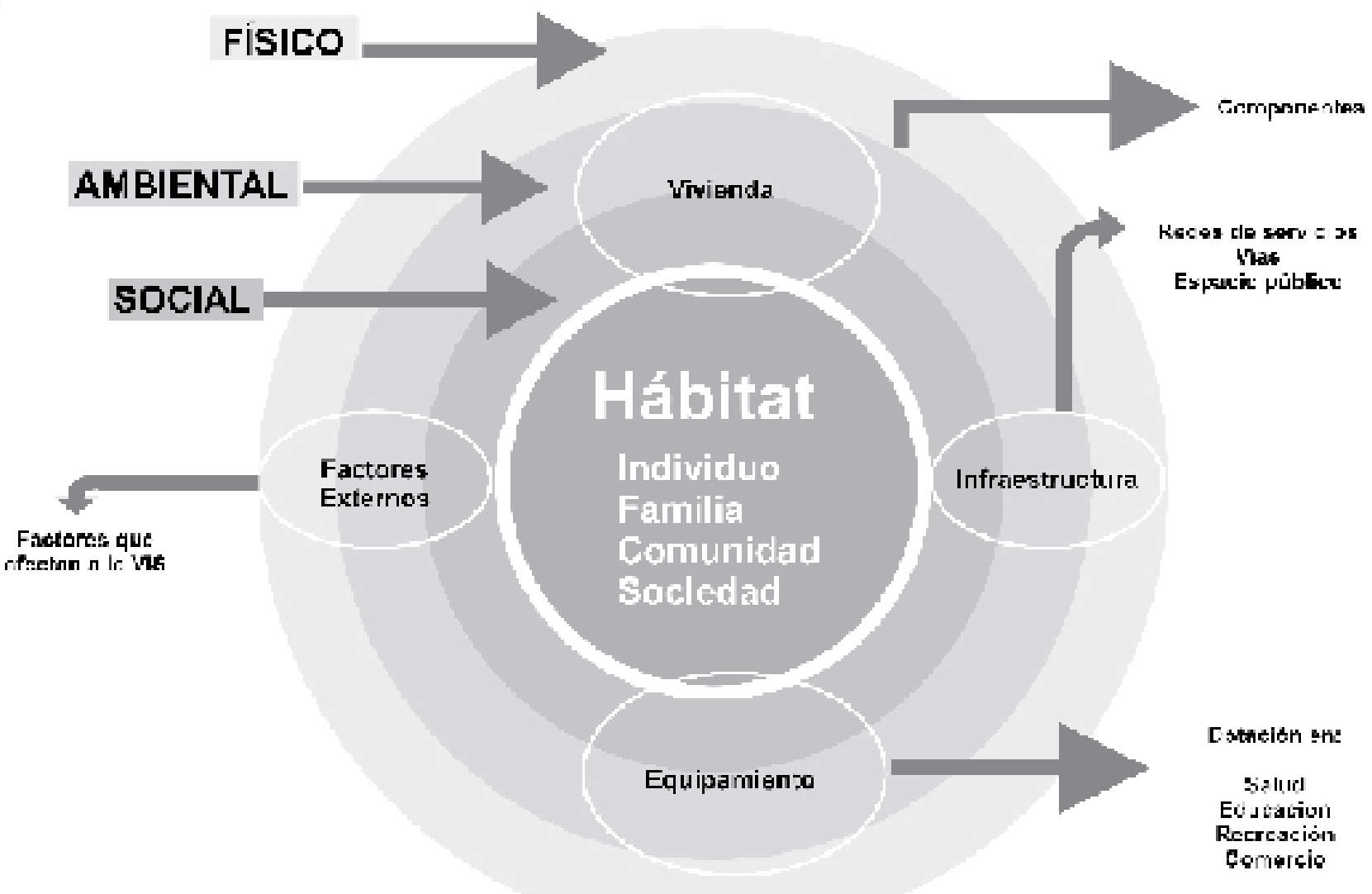

Figura 1. Esquema conceptual de interacción entre los actores en el tema VIS.

\section{Análisis y aportes}

Ver Figura 1

Como resultado del análisis de la problemática relacionada el CENVIS considera en la actualidad a tener en cuenta:

- Es necesario trabajar en las viviendas llamadas "subnormales" como problema central, es decir aquellas que no tienen condiciones mínimas para habitarlas, de manera que podamos generar un alto impacto a través del mejoramiento de vivienda, para lograr un reforzamiento estructural y condiciones de habitabilidad adecuadas, de manera que preservemos la vida y el patrimonio de quienes las habitan.

- Por otra parte, en estas urbanizaciones de origen ilegal es donde se encuentran la mayor cantidad de lotes individuales disponibles para construir y redensificar, de manera que si intervenimos rápidamente con asistencia técnica, microcréditos, provisión de garantías sobre la propiedad del terreno y un modelo de intervención social adecuado, podremos ir construyendo un modelo adecuado de ciudad en donde no existía.
- Una ventaja del trabajo en estas zonas de origen ilegal, es que están conformadas en su mayoría por lotes individuales de 5 o 6 metros de frente por 10 o 12 metros de fondo, lo que hace posible diseñar y desarrollar o mejorar viviendas adecuadas para que la gente viva dignamente.

- En este punto es importante destacar que en este tipo de lotes es viable el desarrollo en altura de viviendas de hasta cuatro pisos, en donde el propietario de la tierra obtiene una utilidad adicional y se está generando suelo nuevo.

- Establecer alianzas estratégicas con los productores de materiales para la creación de los bancos virtuales de materiales.

- Investigación en modelos de apalancamiento financiero exclusivos para estas familias.

- Abrir espacios de investigación y discusión frente a que si bien el problema de la vivienda social es un problema de grandes dimensiones poblacionales, en tanto vincula numerosísimas familias de colombianos, el comienzo de la solución está en el terreno de la gestión pública en cuanto implica la aplicación de los instrumentos propios de la planificación que ya están al alcance de todos los municipios. 
- Diseño y aplicación de modelos de gestión ambiental encaminados a la prevención de riesgos ambientales y de salud pública en las comunidades más vulnerables de nuestra ciudad.

- Las posibilidades de expansión territorial que consagran los POT, da cuenta de importantes ofertas de suelo que puede ser aplicado para vivienda social, luego el problema no es falta de espacio para hacerlo, más tiene que ver con dos factores anexos de gran importancia:

- La propiedad de las tierras, por lo general en manos de pocos propietarios con amplias capacidades financieras, los cuales priorizan planes de alta rentabilidad.

- Las estipulaciones de normas urbanísticas, áreas mínimas de lotes, acceso a la vivienda sólo por vías vehiculares, con sub terrenización de redes eléctricas, exigencias excesivas de estacionamientos, especificaciones técnicas de pavimentos, lo cual encarece la infraestructura y por consiguiente eleva el valor de la vivienda.

\section{Metas}

A fin de lograr una clara consolidación del Centro de Estudios en Vivienda de Interés Social - CENVIS en la Facultad de Ingeniería de Uniminuto se traza un derrotero para:

- Hacer del CENVIS un centro de estudios activo y dinámico al servicio de las familias más vulnerables que desean mejorar o construir su vivienda, elevando así su calidad de vida a través del desarrollo de sus viviendas y en general del hábitat que ocupan.

- Lograr institucionalizar las metodologías, modelos de intervención y estrategias que se construyan, las cuales le permitan al CENVIS ser un operador masivo de las políticas de VIS a nivel nacional.

- Lograr intervenir en espacios institucionales que le permitan a UNIMINUTO - CENVIS aportar todo su conocimiento e incidir y contribuir en la construcción de una verdadera política de hábitat en nuestro país.

\section{Noticias... \\ MENSAJE DEL INGENIERO CARLOS AMAYA (Ex-Decano Facultad de Ingenie- ría, UNIMINUTO)}

Aprovecho esta oportunidad para dirigirme a todos nuestros colaboradores y amigos de UNIMINUTO y de esta Facultad de Ingeniería para agradecerles su apoyo, colaboración y amistad durante los tres años largos en que he tenido el honor de desempeñarme como Decano de Ingeniería, cargo que estoy dejando con muchas satisfacciones y también con experiencias y visión sobre el derrotero de la institución. Aspiro a que encontremos nuevas oportunidades para colaborar con ustedes y con el desarrollo exitoso de esta institución y agradezco muy especialmente la confianza que sus directivos, en particular su Rector General Padre Camilo Bernal, han depositado en mi.

\section{BIENVENIDA}

El Ingeniero NESTOR HUGO MONROY GARCIA Toma la dirección de la Facultad de Ingeniería, de UNIMINUTO. En nombre del comité editorial de está publicación le damos la bienvenida y desde ahora le expresamos nuestro apoyo en este nuevo reto. 